\title{
Measurement of the hadronic cross sections at KLOE with ISR and their impact to the muon anomaly and U-boson search
}

\author{
A.Passeri on behalf oft he KLOE and KLOE-2 Collaborations \\ INFN Sezione Roma Tre \\ Via della Vasca Navale 84, 00146 Roma, Italy \\ E-mail: antonio.passerieroma3.infn.it
}

We present the recent KLOE measurement of the dipion cross section, directly derived from the bin-bybin ratio of $\mathrm{e}^{\mathrm{e}}-\mathrm{e} \rightarrow \pi \pi \gamma$ to $\mathrm{e}+\mathrm{e}^{-} \rightarrow \mu \mu \gamma$ cross sections. The hadronic-loop contribution to the muon anomaly that has been obtained confirms our previous measurements, and the discrepancy between the experimental value of $\mathrm{a}_{\mu}$ and the Standard Model (SM).

With the $\mu \mu \gamma$ sample studied for the dipion cross section, we have obtained a preliminary exclusion plot for the U-boson in the Dark Force sector, in the mass range from $600-1000 \mathrm{MeV}$ that is presented together with the excluded region from the analysis of the Dalitz decays, $\varphi \rightarrow \eta e^{+} \mathrm{e}^{-}$.

International Conference on the Structure and the Interactions of the Photon including the 20th International Workshop on Photon-Photon Collisions and the International Workshop on High Energy Photon Linear Colliders

May 20-24, 2013

Paris, France 


\section{Introduction}

Measurements of the muon magnetic anomaly at the Brookhaven Laboratory have reached a fractional accuracy of $0.5410^{-6}, \mathrm{a}_{\mu}=\left(\begin{array}{ll}11 & 659 \\ 208.0 \pm 6.3\end{array}\right) \times 10^{-10}$ [1]. The result differs from the Standard Model prediction by 3.2-3.6 standard deviations[2, 3, 4, 5]. The main source of uncertainty on the SM value of $a_{\mu}$ is the leading hadronic vacuum polarization term. It is obtained from a dispersion integral [6] over the "bare" cross section $\sigma^{0}\left(\mathrm{e}+\mathrm{e}^{-} \rightarrow\right.$ hadrons $\left.(\gamma)\right)$ that is derived from the physical cross section, inclusive of final state radiation, removing vacuum polarization (VP) and contributions due to additional photon emission in the initial state. The leading order hadronic contribution is $\sim 690 \times 10^{-10}$ and the $\mathrm{e}+\mathrm{e}^{-} \rightarrow \pi+\pi-(\gamma)$ process measured by KLOE contributes to $75 \%$ of the value and $40 \%$ of the uncertainty. The KLOE experiment at the DAФNE $\varphi$-factory in Frascati, was the first to exploit Initial State Radiation (ISR) processes for the precision measurement of the hadronic cross section below $1 \mathrm{GeV}$. In 2005 and 2008 KLOE published two measurements of the $\mathrm{e}+\mathrm{e}^{-} \rightarrow \pi \pi \gamma$ cross section, with the ISR photon at small angle[7, 8]. An independent measurement with the photon emitted at large angle, to reach the dipion production threshold at $s=0.1 \mathrm{GeV}^{2}$, was published in year 2011[9]. The three measurements of $\sigma\left(\mathrm{e}+\mathrm{e}^{-} \rightarrow \pi+\pi-\right)$ cover the interval $\left[0.1<\mathrm{M}_{\pi \pi}^{2}<0.95\right] \mathrm{GeV}^{2}$, with consistent results and a combined fractional uncertainty of about $1 \%$. This paper reports on a more recent analysis of KLOE data, which directly derives the pion form factor from the bin-by-bin ratio of $\mathrm{e}^{+} \mathrm{e}^{-} \rightarrow \pi \pi \gamma$ to $\mathrm{e}^{-} \mathrm{e}^{-} \rightarrow \mu \mu \gamma$ cross sections[10].

\section{Measurement of $\sigma\left(e^{+} e^{-} \rightarrow \pi^{+} \pi^{-}\right)$}

The differential ISR cross section for the $\mathrm{e}+\mathrm{e}^{-} \rightarrow \pi+\pi-\gamma$ final state is related to the dipion cross section $\sigma_{\pi \pi} \equiv \sigma\left(\mathrm{e}+\mathrm{e}^{-} \rightarrow \pi+\pi-\gamma\right)$

$$
\left.s \frac{d \sigma\left(\pi^{+} \pi^{-} \gamma\right)}{d s_{\pi}}\right|_{I S R}=\sigma_{\pi \pi}\left(s_{\pi}\right) H\left(s_{\pi}, s\right)
$$

where the radiator function $\mathrm{H}$ is computed from QED with complete NLO corrections[12]. Equation 1 is also valid for the dimuon final state with the same radiator function $\mathrm{H}$. We can therefore determine $\sigma_{\pi \pi}$ from the ratio of the $\mathrm{e}^{\mathrm{e}} \mathrm{e}^{-} \rightarrow \pi+\pi-\gamma$ and $\mathrm{e}+\mathrm{e}^{-} \rightarrow \mu+\mu-\gamma$ differential cross sections:

$$
\sigma^{0}\left(\pi^{+} \pi^{-}, s^{\prime}\right)=\frac{d \sigma\left(\pi^{+} \pi^{-}, I S R\right) / d s^{\prime}}{d \sigma\left(\mu^{+} \mu^{-}, I S R\right) / d s^{\prime}} \times \sigma^{0}\left(e^{+} e^{-} \rightarrow \mu^{+} \mu^{-}, s^{\prime}\right)
$$

Final state photon emission for both $\pi+\pi-\gamma$, and $\mu+\mu-\gamma$ channels slightly modifies Eq.2, and it has been considered in our analysis[13], where only events with photon emitted at small angle are used, as discussed in Refs.[7, 8], a choice that results in a large enhancement of ISR with respect to the FSR contribution. The ratio method has several advantages: (i) the H function does not appear in Eq.2 so that the measurement of $\sigma_{\pi \pi}$ is not affected by the related systematic uncertainty of $0.5 \%$;(ii) using the same data sample for the $\pi+\pi-\gamma$ and $\mu+\mu-\gamma$ events there is no need for luminosity measurements; (iii) vacuum polarization corrections and most of 
the other radiative corrections cancel in the ratio; (iv) using the same fiducial volume, acceptance corrections to the $\pi+\pi-\gamma$ and $\mu+\mu-\gamma$ spectra almost cancel resulting in a small systematic uncertainty. The pion form factor and $\Delta^{\pi \pi} a_{\mu}$ have been obtained using the $\pi \pi \gamma$ differential cross section of Ref.[8] and the precision measurement of $d \sigma \mu \mu / \mathrm{ds} \mu$, discussed in the following section.

\subsection{The $e^{+} e^{-} \rightarrow \mu^{+} \mu^{-} \gamma$ cross section}

The data sample used in this analysis corresponds to an integrated luminosity of $239.2 \mathrm{pb}^{-1}$ collected in 2002, with low machine background and stable DAФNE conditions. The KLOE detector has been described in details elsewhere [11]. The $\mu^{+} \mu^{-} \gamma$ event selection is based on the following requirements:

(1) Events must have at least two tracks of opposite sign, with origin at the interaction point and polar angle satisfying $50^{\circ}<\theta<130^{\circ}$. The reconstructed momenta must satisfy $p_{\perp}>$ $160 \mathrm{MeV}$ or $|p z|>90 \mathrm{MeV}$, to ensure good reconstruction and efficiency.

(2) The polar angle $\theta_{\mu \mu}$ of the dimuon system obtained from the momentum of the two tracks $\left(\mathbf{p}_{\mu \mu}=\mathbf{p}^{+}+\mathbf{p}\right)$ must satisfy $\left|\cos \theta_{\mu \mu}\right|>\cos \left(15^{\circ}\right)$.

(3) A particle identification estimator is defined for each track, $L \pm$, using time-of-flight information and the value and shape of the energy deposit of each charged particle in the calorimeter. Events with both tracks having $L_{ \pm}<0$ are identified as eev events and rejected. The loss of signal events due to this cut is less than $0.05 \%$, as evaluated with $\mu \mu$ samples, obtained from both data and Monte Carlo events.

(4) The computed mass for the two observed particles must satisfy $80<m_{x}<115 \mathrm{MeV}$ as shown in Fig.1 left.
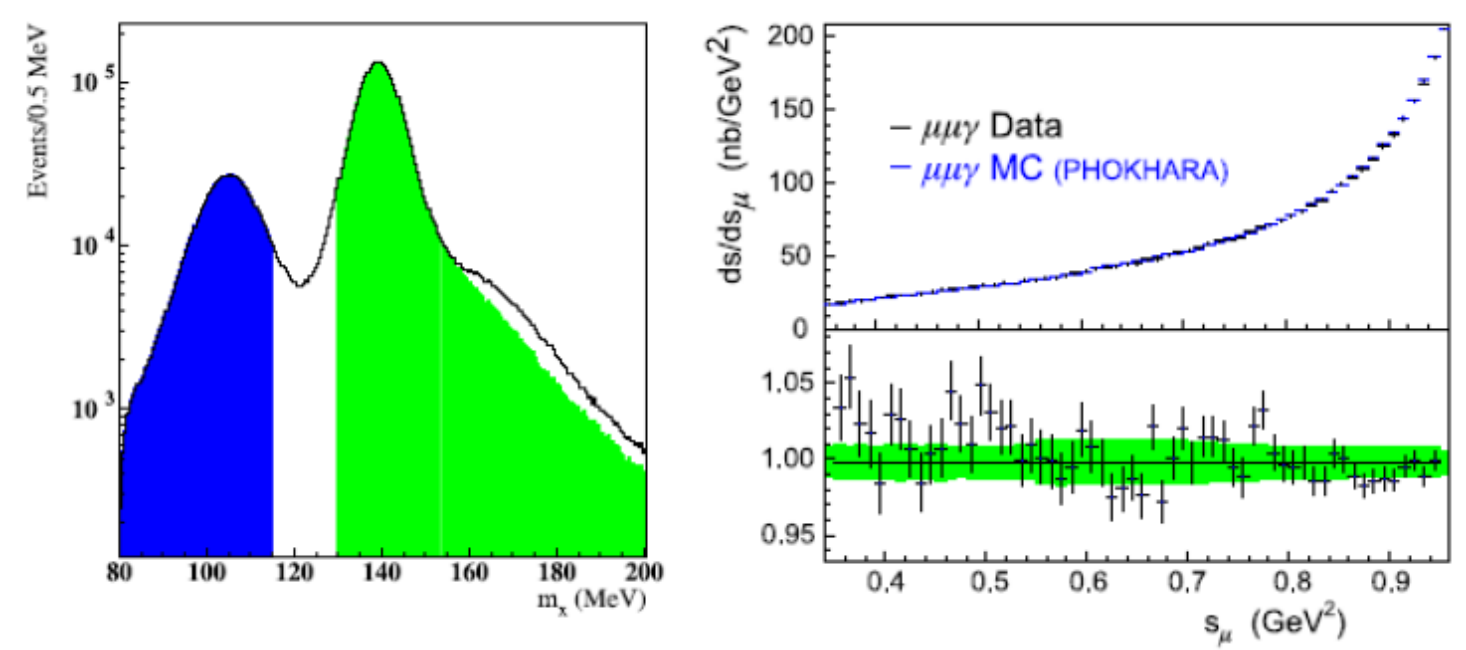

Fig. 1. Left: data $\mu \mu \gamma$ and $\pi \pi \gamma$ regions in the $m_{x}$ spectrum. The $\mu \mu \gamma$ and $\pi \pi \gamma$ accepted regions are shown in blue and green respectively. A residual contamination of $\pi^{+} \pi^{-} \pi^{0}$ events is visible at high $m_{x}$ values. Righttop: Comparison of data and MC results for $\mathrm{d} \sigma_{\mu \mu} / \mathrm{ds}_{\mu}$. Right-bottom: Ratio of the two spectra. The band shows systematic errors.

Residual $\mathrm{e}^{+} \mathrm{e}^{-} \gamma, \pi^{+} \pi^{-} \gamma$ and $\pi^{+} \pi^{-} \pi^{0}$ backgrounds are evaluated by fitting the observed $\mathrm{m}_{\mathrm{x}}$ spectrum with a superposition of Monte Carlo simulation (MC) distributions describing signal 
and $\pi^{+} \pi^{-} \gamma, \pi^{+} \pi^{-} \pi^{0}$ backgrounds, and a distribution obtained from data for the $\mathrm{e}^{+} \mathrm{e}^{-} \gamma$ background. In the $\rho$ mass region, the fractional $\pi^{+} \pi^{-} \gamma$ yield in the $\mu \mu \gamma$ acceptance region is about $15 \%$ of the sample. To improve the MC description of the low-energy $\mathrm{m}_{\mathrm{x}}$ tail of $\pi^{+} \pi^{-} \gamma$ events in the muon peak, we apply a data/MC resolution correction, function of $s_{\mu}$, using a control sample of $\varphi \rightarrow$ $\pi^{+} \pi^{-} \pi^{0}$ events.

Contributions from $\mathrm{e}^{+} \mathrm{e}^{-} \rightarrow \mathrm{e}^{+} \mathrm{e}^{-} \mu^{+} \mu^{-}$and $\mathrm{e}^{+} \mathrm{e}^{-} \rightarrow \mathrm{e}^{+} \mathrm{e}^{-} \pi^{+} \pi^{-}$processes are evaluated using the Nextcalibur[13] and Ekhara[14] MC generators. Systematic errors in the background subtraction include: (i) errors on the parameters from the fit procedure: these decrease monotonically from $0.7 \%$ to $0.1 \%$ with respect to $\mathrm{s}_{\mu}$; (ii) the uncertainty on the data/MC resolution corrections: about $1 \%$ in the $\rho$ mass region, smaller at higher $s \mu$, negligible at lower $\mathrm{s}_{\mu}$ values; (iii) the uncertainty on the $\mathrm{e}^{+} \mathrm{e}^{-} \rightarrow \mathrm{e}^{+} \mathrm{e}^{-} \mu^{+} \mu^{-}$process: about $0.4 \%$ at low $\mathrm{s}_{\mu}$, rapidly falling to $0.1 \%$ for $s \mu>0.5 \mathrm{GeV}^{2}$. The correctness of the background estimate has been checked by two independent methods. With the first, we perform a kinematic fit of the two-track events assuming a $\mu \mu \gamma$ state. The $\chi^{2}$ value obtained is taken as discriminant variable, instead of $m_{x}$, and used in the fitting procedure described above; with the second, we improve the $\pi$ - $\mu$ separation applying a quality cut on the helix fit for both tracks. This cut reduces the dipion background in the dimuon signal region by more than a factor of two. The background fractions obtained for both cases are in good agreement with the standard procedure. The differential $\mu^{+} \mu^{-} \gamma$ cross section is obtained from the observed event count $\mathrm{N}_{\mathrm{obs}}$ and background estimate $\mathrm{N}_{\mathrm{bkg}}$ :

$$
\frac{d \sigma_{\mu \mu \gamma}}{d s_{\mu}}=\frac{N_{o b s}-N_{b k g}}{\Delta s_{\mu}} \frac{1}{\varepsilon\left(s_{\mu}\right) \mathcal{L}}
$$

where $\mathcal{L}$ is the integrated luminosity from Ref.[15] and $\varepsilon\left(\mathrm{s}_{\mu}\right)$ the selection efficiency. Figure 1, right-top, shows the measured $\mu^{+} \mu^{-} \gamma$ cross section compared with the QED calculations to NLO, using the MC code Phokhara[11]. Figure 1, right-bottom, shows the ratio between the two differential cross sections. The band indicates the systematic uncertainty, experimental and theoretical.

\section{The hadronic vacuum contribution to $a_{\mu}$}

From the bin-by-bin ratio between our published[8] $\pi^{+} \pi^{-} \gamma$, and the $\mu^{+} \mu^{-} \gamma$ differential cross sections, we obtain the bare cross section $\sigma_{\pi \pi(\gamma)}^{0}$ (inclusive of FSR, with VP effects removed) which is used in the dispersion integral for computing $\Delta^{\pi \pi}{ }_{\text {a }}$. Figure 2 shows the $\pi^{+} \pi^{-} \gamma$ and $\mu^{+} \mu^{-} \gamma$ event spectra after background subtraction and data/MC corrections (left) and the bare cross section $\sigma_{\pi \pi(\gamma)}^{0}$ (right). Systematic uncertainties on $\sigma_{\pi \pi(\gamma)}^{0}$ are smaller than the individual uncertainty on $\sigma\left(\mathrm{e}^{+} \mathrm{e}^{-} \rightarrow \pi \pi \gamma\right)$ and $\sigma\left(\mathrm{e}^{+} \mathrm{e}^{-} \rightarrow \mu \mu \gamma\right)$ due to correlation between the two measurements[12].

The dispersion integral gives $\Delta^{\pi \pi}{ }_{\mathrm{a} \mu}=\left(385.1 \pm 1.1_{\mathrm{stat}} \pm 2.6_{\mathrm{exp}} \pm 0.8_{\mathrm{th}}\right) \times 10^{-10}$ in the interval 0.35 $<\mathrm{M}_{\pi \pi}^{2}<0.95 \mathrm{GeV}^{2}$, that is consistent with our previous measurements. This result, with comparable total experimental uncertainty and a theoretical error reduced by about $70 \%$, confirms the current discrepancy between the SM prediction and the experimental value of $a_{\mu}$. 

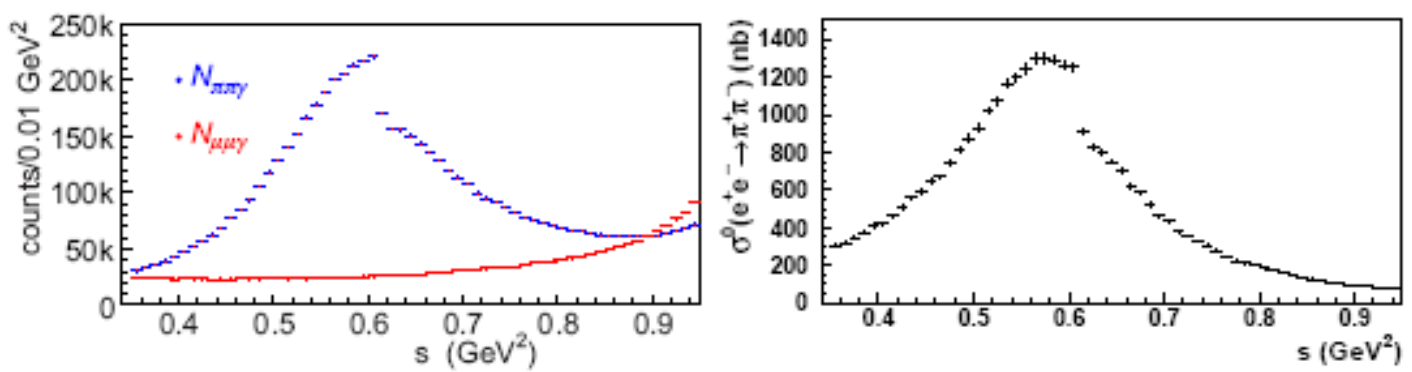

Fig. 2. Square-invariant-mass distributions of $\pi^{+} \pi^{-} \gamma$ (higher counts from $0-0.9 \mathrm{GeV}^{2}$ ) and $\mu^{+} \mu^{-} \gamma$ (lower counts from $0-0.9 \mathrm{GeV}^{2}$ ) events after background subtraction and data/MC corrections (left); the bare cross section from the $\pi^{+} \pi^{-} \gamma / \mu^{+} \mu^{-} \gamma$ ratio (right).

\section{Searches for the U-boson}

Some models of physics beyond the SM predict the existence of light neutral vector particles (called U-bosons) mediator of new gauge interactions under which ordinary matter is uncharged[16]. Motivated by astrophysical arguments, their mass, $\mathbf{M}_{U}$, is expected to be of order of $1 \mathrm{GeV}$ or lighter[17, 18]. Coupling of SM particles with the $\mathrm{U}$ is possible via kinetic mixing between the $U$ and the ordinary photon, regulated by a dimensionless parameter $\varepsilon$, expected to be of order $\varepsilon^{\sim} 10^{-3}$ or lower. High-luminosity $\mathrm{e}^{+} \mathrm{e}^{-}$colliders at the $\mathrm{GeV}$ scale have been recognized to be an ideal environment to search for the U-boson in the Dark Force sector. These new particles can be observed as sharp resonances at $\mathrm{M}_{U}$ in the invariant mass distribution of charged lepton or pion pairs in reactions of the type e+e- $\rightarrow 1^{+} 1^{-} \gamma$ or $\mathrm{V} \rightarrow \mathrm{Pl}^{+} 1^{-}$, where $\mathrm{V}(\mathrm{P})$ stands for any vector (pseudoscalar) meson, and $\mathrm{l}^{ \pm}$can be muons, electrons or charged pions.

KLOE has searched for $\mathrm{U}$ boson production in both modes, using $\varphi \rightarrow \eta \mathrm{e}^{+} \mathrm{e}^{-}$events (a), and $\mathrm{e}^{+} \mathrm{e}^{-} \rightarrow \mu^{+} \mu^{-} \gamma$ events (b). As for reactions (a), a first paper has been published[19] in which the presence of the $\eta$ meson was tagged using its $\pi^{+} \pi^{-} \pi^{0}$ decays; a second paper has been subsequently issued[20] in which also the $3 \pi^{0}$ decay channel of the $\eta$ was used. In both cases a sample corresponding to $1.7 \mathrm{fb}^{-1}$ of data at the $\varphi$ peak was used; no evidence of the $\mathrm{U}$ boson is found, and the exclusion plot, in the interval $30<\mathrm{M}_{\mathrm{U}}<400 \mathrm{MeV}$, has been obtained (Fig.3). Reaction (b) was studied on the sample used for the measurement of the ratio, $\mathrm{R}=\sigma\left(\mathrm{e}^{+} \mathrm{e}^{-} \rightarrow\right.$ $\left.\pi^{+} \pi^{-}(\gamma)\right) / \sigma\left(\mathrm{e}^{+} \mathrm{e}^{-} \rightarrow \mu^{+} \mu^{-}(\gamma)\right)$, exploiting the precision MC simulation of the QED process $\mathrm{e}^{+} \mathrm{e}^{-} \rightarrow$ $\mu \mu \gamma$ (Fig.1). The exclusion plot is obtained using the CLS technique. The preliminary result shown in Fig. 3 covers the mass region $600<\mathrm{M}_{\mathrm{U}}<1000 \mathrm{MeV}$ and is currently being extended to $500 \mathrm{MeV}$. 


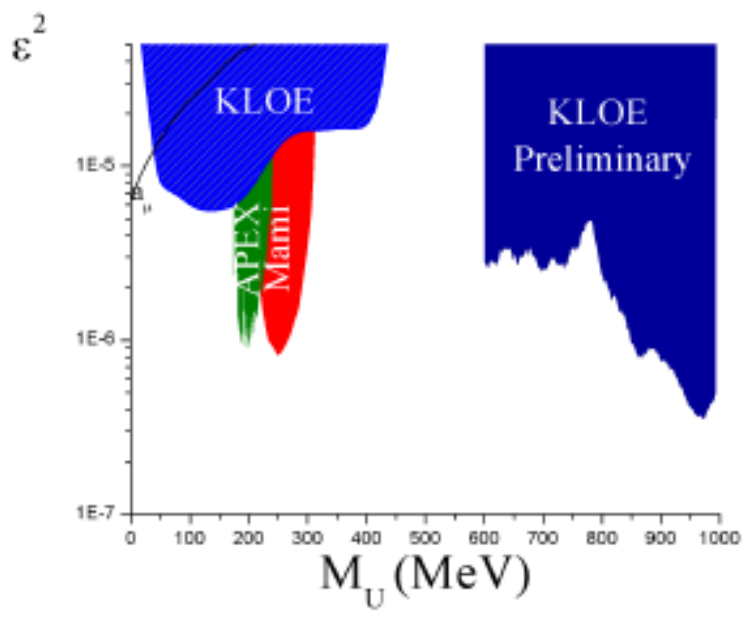

Fig. 3. Preliminary KLOE-2 exclusion plot in the $M_{U}-\varepsilon^{2}$ plane (90\% C.L.). Results are shown for the $\varphi \rightarrow$ $\eta \mathrm{e}^{+} \mathrm{e}^{-}$analyses (left) and for $\mathrm{e}^{+} \mathrm{e}^{-} \rightarrow \mu^{+} \mu^{-} \gamma$ (right). The results from the APEX and MAMI-A1 experiments are also shown.

\section{References}

[1] G.W. Bennett et al. (Muon g-2), Phys. Rev. D73, 072003 (2006)

[2] J.P. Miller, E. de Rafael, B.L. Roberts, Rept.Prog.Phys. 70, 795 (2007)

[3] F. Jegerlehner, A. Nyffeler, Phys.Rept. 477, 1 (2009)

[4] M. Davier, A. Hoecker, B. Malaescu, Z. Zhang, Eur.Phys.J. C71, 1515 (2011)

[5] K. Hagiwara et al., J.Phys. G38, 085003 (2011)

[6] B. Lautrup, A. Peterman, E. De Rafael, Nuovo Cim. A1, 238 (1971)

[7] A. Aloisio et al. (KLOE), Phys.Lett. B606, 12 (2005)

[8] F. Ambrosino et al. (KLOE), Phys. Lett. B670, 285 (2009)

[9] F. Ambrosino et al. (KLOE), Phys.Lett. B700, 102 (2011)

[10] D. Babusci et al. (KLOE), Phys.Lett. B720, 336 (2013)

[11] M. Adinolfi, et al., Nucl. Instrum. Meth. A 488 (2002) 51; M. Adinolfi, et al., Nucl. Instrum. Meth. A 482 (2002) 364; M. Adinolfi, et al., Nucl. Instrum. Meth. A 492 (2002) 134.

[12] H. Czyz, A. Grzelinska, J.H. Kuhn, G. Rodrigo, Eur. Phys. J. C39, 411 (2005)

[13] P. Lukin et al., http://www. Inf.infn.it/kloe2/tools/getfile.php?doc fname $=\mathrm{K} 2 \mathrm{PD}-6 \cdot \mathrm{pdf} \& \mathrm{doc}$ ftype $=$ docs

[14] F.A. Berends et al., Comput. Phys. Commun. 136, 148 (2001)

[15] H. Czyz, E. Nowak-Kubat, Phys. Lett. B634, 493 (2006)

[16] F. Ambrosino et al. (KLOE), Eur. Phys. J. C47, 589 (2006)

[17] P. Fayet, Phys. Lett. B95, 285 (1980) 
[18] N. Arkani-Hamed, N. Weiner, JHEP 12, 104 (2008)

[19] M. Pospelov, A. Ritz, M.B. Voloshin, Phys. Lett. B662, 53 (2008)

[20] F. Archilli et al., Phys. Lett. B706, 251 (2012)

[21] D. Babusci et al. (KLOE-2), Phys. Lett. B720, 111 (2013) 